\title{
An opponent-process interpretation of postshock bursts in appetitive responding
}

\author{
J. D. LaBARBERA and WILLIAM F. CAUL \\ Vanderbilt University, Nashville, Tennessee 37240
}

\begin{abstract}
While eight food-deprived rats pressed a lever for food during daily 1-h sessions, four CS-Shock $_{2}$ trials were presented. Trials were preceded by either a Shock of $_{1} .25, .50, .75 \mathrm{~mA}$ or no Shock $_{1}$. It was found that the rate of appetitive responding during the CS was greater on Shock ${ }_{1}-\mathrm{CS}$ Shock $_{2}$ trials relative to CS-Shock ${ }_{2}$ trials. The data indicate that the phenomenon of postshock bursts in responding can occur in the absence of a signal for safety after shock. The results conform to the predictions of the opponent-process theory of motivation.
\end{abstract}

A number of investigators have found that in a free-responding situation, an animal may exhibit a burst of responses after an aversive event. When an animal presses a lever on a Sidman avoidance schedule, it may emit a burst of responses following each shock (e.g., Sidman, 1958). Church and Getty (1972) point out that, although the notion has not been seriously tested, these bursts on an avoidance baseline may involve some form of reinforcement, such as delay of onset of the next shock.

Postshock bursts in appetitive responding have also been noted. Estes and Skinner (1941) found that rats that pressed a lever for food exhibited bursts in responses at the termination of a response-suppressing stimulus and shock. They suggested that the animals were "compensating" for the suppression in responding that occurred during the signal, but they did not attempt to explain the mechanism by which such compensation might occur. Church, Wooten, and Matthews (1970) trained rats to press for food and then presented shocks on a 2-min random schedule. They found that shock reduced the overall rate of appetitive behavior, but that a given shock often led to a local flurry of responses. Weiss and Strongman (1969) also presented shocks to rats that had been trained to barpress for food. The shocks occurred randomly on a 2-min schedule during a 3-min flashing light warning signal, rather than at its termination as in the conventional conditioned suppression procedure. They found that the initial effect of shock was to suppress responding generally and to cause short bursts of postshock responding.

Several explanations have been proposed to account for postshock bursts in appetitive responding. Church and Getty (1972) suggest that, if a response is inhibited,

A version of this study was presented at the annual meeting of the Southeastern Psychological Association in New Orleans, March 1976. Requests for reprints should be sent to J. D. LaBarbera, Department of Psychology, Vanderbilt University, Nashville, Tennessee 37240. the occurrence of shock or any other intense stimulus may be sufficient to disinhibit the response. Alternatively, postshock bursts may comprise a reflexive reaction to shock, such as an innate species-specific defense reaction (Bolles, 1970) or a stereotyped attack response (Weiss \& Strongman, 1969) similar to that described by Ulrich and Azrin (1962). Consistent with this explanation, Myer states that: "It seems possible that the effect of moderate pain is to potentiate any 'active' behavior which has a high probability of otcurrence in the situation" (Myer, 1971, p. 505).

Davis (1970) argues that postshock bursts in appetitive responding may be due to the presence after shock of a reliable signal for safety, which serves to reduce response-inhibiting fear. He notes that, in the Weiss and Strongman study, subjects that were exposed to a VI 1-min food schedule were generally suppressed during the 3-min warning signal, and therefore were "cost" an average of three pellets. Because shocks during the warning signal were programmed to occur on a random schedule with a mean interval of $2 \mathrm{~min}$, the "safest" time during the otherwise unsafe warning signal was immediately after shock, when on the average another shock was not scheduled to occur for $2 \mathrm{~min}$. He maintains that Weiss and Strongman set up an "if shock then no shock" contingency, whereby shock was a discriminative stimulus for safety.

The question remains, however, as to precisely how this view applies to the Weiss and Strongman situation. While a safety signal explanation may account for postshock bursts in situations in which the termination of shock reliably forecasts a period free from approaching shocks (e.g., Estes \& Skinner, 1941), it does not seem readily capable of accounting for the phenomenon in either the Church et al. (1970) or the Weiss and Strongman (1969) studies, where random shock schedules were employed. Davis' interpretation that postshock bursts result from the presence of a fear-inhibiting period of 
safety after shock rests on assumptions about the nature of the actual shock schedule used and/or assumptions about the subject's conception of this schedule. Because shocks were delivered by Weiss and Strongman on a temporally random schedule, the period immediately after shock had the same probability of shock delivery as any other period during the warning signal, and a given shock provided no information regarding the absence of forthcoming shocks. Therefore, since the level of conditioned fear is related to the instantaneous probability of an aversive event (Rescorla, 1968), the warning signal should be characterized by a constant level of fear, i.e., no decrement in fear immediately after shock. It may have been the case, however, that the animals initially learned that shock termination signaled safety if none of the first few shocks were immediately followed by another shock. Consistent with this artifactual explanation is Weiss and Strongman's finding that the bursts in their study disappeared after about five 1-h sessions.

In order to explain these data with a safety-signal analysis, one may have to include the notion that subjects concentrate their responses after shock as a result of a mistaken conception of the distribution of shocks in time (Church et al., 1970), i.e., if a shock has recently occurred, they may act as if another shock was not immediately forthcoming. Before this notion is pursued, however, it seems important to explore other mechanisms by which response bursts might occur.

The primary purpose of the present study was to assess the presence of postshock bursts in appetitive responding in a manner that rules out an explanation based upon the operation of a reliable signal for safety in the aftermath of a shock. One way of preventing an increment in postshock responding due to the presence of a signal for safety is to employ a random schedule of aversive events. A second tack, and the one followed here, would be to equalize the amount of safety that is assumed to exist on two kinds of trials by presenting a CS for shock in both cases, but where the CS is sometimes preceded by a shock and sometimes not. The safety signal hypothesis predicts that there should be no difference in fear on the two trials and, therefore, no difference in appetitive responding. This second technique may have some advantage in demonstrating that postshock bursts comprise a reaction to the previous shock and not an anticipation of the next shock. First, this method is not affected by the subject's conception of randomness, and second, it rules out the operation of the safety signal due to a subject's initial and temporary absence of experience with shock in the immediate aftermath of shock.

\section{METHOD}

\section{Subjects}

The subjects were eight male Charles River $C D$ rats. The age of each subject was 77 days, and the mean weight was $272 \mathrm{~g}$ at the beginning of pretraining.

\begin{abstract}
Apparatus
Two identical lever boxes (22.5 × $10 \times 22.5 \mathrm{~cm}$ high) were employed. The roof, back panel, and two side panels were made of white, opaque Plexiglas; the front panel was transparent Plexiglas. The lever box floor was composed of parallel stainless steel bars that were $1.2 \mathrm{~cm}$ in diameter and spaced $1.8 \mathrm{~cm}$ apart. Scrambled shocks were delivered to the grid. A stainless steel lever extended $1.5 \mathrm{~cm}$ through the side panel, $3.8 \mathrm{~cm}$ above the floor. A Davis pellet dispenser delivered 45-mg Noyes Precision food pellets through an opening in the side panel next to the lever. Each lever box was housed in a large insulation-board chamber designed to attenuate sounds and block visual stimuli. Attached to the interior roof of the insulating chamber were a $71 / 2-W$ houselight and a speaker to deliver the CS and white noise. Control and response monitoring equipment was located in an adjacent room.
\end{abstract}

\section{Procedure}

On Day 1, each animal was weighed and all food was removed. For the duration of the experiment, each animal was weighed and given enough ground Purina Lab Chow in its home cage to maintain its Day 1 weight. Since the animals had not attained their adult weights by Day 1 , this procedure constituted partial food deprivation.

On Days 11-17, the animals were taken two at a time from their home cages and each placed in one of the lever boxes, where food pellets were available on a CRF schedule. Sessions lasted for $30 \mathrm{~min}$ each day. On Days 18-27, a VI 1-min schedule of food reinforcement was instituted, and session length was increased to $60 \mathrm{~min}$.

Testing began on Day 28. The subjects were taken two at a time from their home cages and placed in either lever box where food continued to be available on a VI 1-min schedule. In addition, each subject received four CS-Shock ${ }_{2}$ trials while in the appetitive situation. Each trial consisted of a $70-\mathrm{dB}, 1-\mathrm{kHz}$ tone $\mathrm{CS}$ of $30 \mathrm{sec}$ duration followed by Shock $_{2}(.75-\mathrm{mA}$ intensity and $.5-\mathrm{sec}$ duration). On three CS-Shock ${ }_{2}$ trials in a session, the CS was preceded by Shock 1.5 -sec duration), which terminated with the onset of the CS. Shock, intensity was $.25, .50$, or $.75 \mathrm{~mA}$. For the fourth CS-Shock ${ }_{2}$ trial, no shock occurred prior to the CS. During each 1 -h session, trials were scheduled 14,15 , or 16 min apart, and the sequence of the four different trials varied from session to session. During these sessions, leverpressing was monitored during the 30 -sec CS by a set of six sequential 5 -sec counters. Testing continued for 20 sessions.

\section{RESULTS}

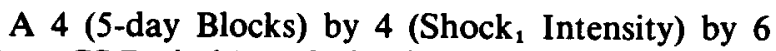
(5-sec CS Periods) analysis of variance was computed to evaluate the leverpressing data during the testing phase of the experiment. The analysis showed a significant CS Periods main effect $[F(5,7)=11.481$, $\mathrm{p}<.001$ ]. As depicted in Figure 1, responses were concentrated at the beginning of the 30-sec CS and decreased by the second 5 -sec interval for all four intensity levels of Shock. . The Shock $_{1}$ Intensity main effect was also significant $[\mathrm{F}(3,7)=8.528, \mathrm{p}<.001]$. 


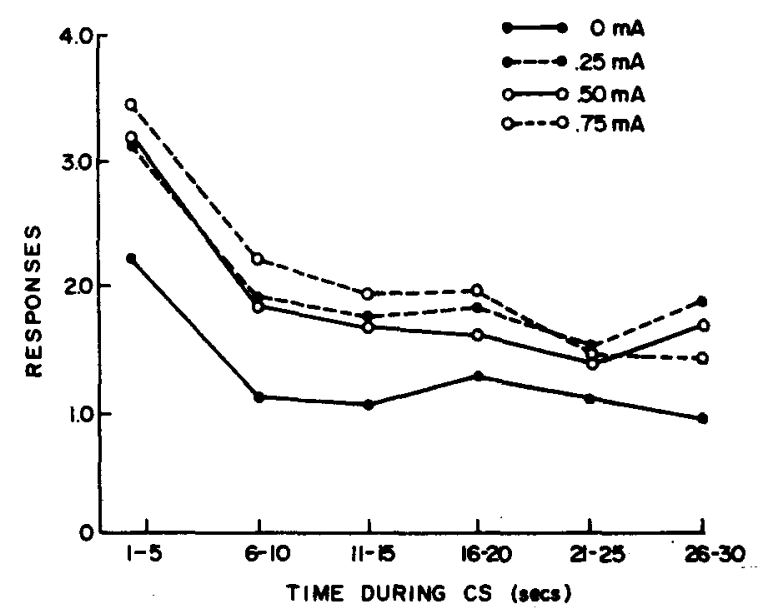

Figure 1. Mean number of responses emitted during 5-sec segments of the 30-sec CS when preceded by a Shock ${ }_{1}$ of $0, .25, .50$, or $.75 \mathrm{~mA}$. The means include data from all 20 days of testing.

Pairwise comparisons showed that subjects responded more during the CS when a Shock was $^{2} .25 \mathrm{~mA}$ $[F(1,7)=49.311, \mathrm{p}<.001], .50 \mathrm{~mA}[\mathrm{~F}(1,7)=$ $11.383, \mathrm{p}=.01]$, or $.75 \mathrm{~mA}[\mathrm{~F}(1,7)=11.338$, $\mathrm{p}<.05$ ] than when Shock $_{1}$ was absent. Figure 1 shows that the response gradients for trials on which Shock $_{1}$ was $.25, .50$, or $.75 \mathrm{~mA}$ were elevated over that for trials on which Shock ${ }_{1}$ was absent. The remaining possible pairwise comparisons revealed no significant differences in responding during the CS for the various levels of Shock . $_{1}$

Figure 1 also shows that the difference between rates of responding for trials when Shock ${ }_{1}$ was .25, .50 , or $.75 \mathrm{~mA}$ and when Shock $_{1}$ was absent remained fairly stable throughout the 30 -sec CS, i.e., the Shock, Intensity by CS Periods interaction was nonsignificant $[F(3,7)<1]$.

Inspection of Figure 2 suggests that the rate of responding during CS increased over days differentially as a function of Shock ${ }_{1}$ intensity. This interaction in the overall analysis, which included the asymptotic performance during the final two 5-day blocks, however, was not significant $[F(3,7)=1.05$, $\mathrm{p}=.40]$. An analysis of response rate that included only the first two 5-day blocks showed the 5-day Blocks by Shock, Intensity interaction to be significant $[F(3,7)=3.04, p<.05]$ : Pairwise compari-

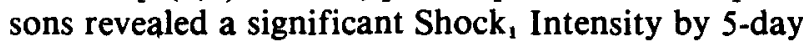
Blocks interaction for Shock $1=.75 \mathrm{~mA}$ relative to when Shock, was absent $[F(1,7)=8.638, p<.05]$. The remaining comparisons were nonsignificant. These results indicate that the groups defined by the extreme $\mathrm{US}_{1}$ values, i.e., $.75 \mathrm{~mA}$ vs. $0 \mathrm{~mA}$, did differ reliably in rate of responding over days.

Analyses were also performed to evaluate the rates of responding during the first two 5 -sec periods of the CS over the first 10 days of testing. A 2 (5-day
Blocks) by 4 (Shock 1 Intensity) analysis of variance showed a significant 5-day Blocks by Shock ${ }_{1}$ Intensity interaction $[F(3,21)=3.956, p<.05]$. Pairwise comparisons indicated a significant 5 -day Blocks by Shock $_{1}$ Intensity interaction for Shock ${ }_{1}=.75 \mathrm{~mA}$ relative to when Shock $_{1}$ was absent $[F(1,7)=$ 7.706, $p<.05$ ], implying that the magnitude of the increase in responding on trials preceded by Shock $_{1}=.75 \mathrm{~mA}$ relative to trials on which Shock was absent increased over days.

\section{DISCUSSION}

The results indicate that bursts in appetitive responding can occur in the aftermath of an aversive event and that the increment in responding can last for as long as $30 \mathrm{sec}$ after the aversive event. When CS-Shock $_{2}$ trials were preceded by a Shock ${ }_{1}$, the animals exhibited a higher rate of responding during the CS than when Shock, was absent.

A possible explanation for the finding of higher rates of responding during the CS for trials preceded by Shock ${ }_{1}$ is that Shock $_{1}$ served as a discriminative cue for distinguishing between the initial (safe) and latter (unsafe) parts of the CS. The higher rates of responding during the initial seconds of the preshocked trials may conceivably have reflected a subject's increased anticipation of safety. However, such an associationistic argument does not seem readily capable of accounting for the higher rates of responding for preshocked trials during the seconds immediately prior to Shock $_{2}$.

A nonassociative explanation would hold that the postshock increment in responding represented not a subject's anticipation of forthcoming Shock ${ }_{2}$, but a

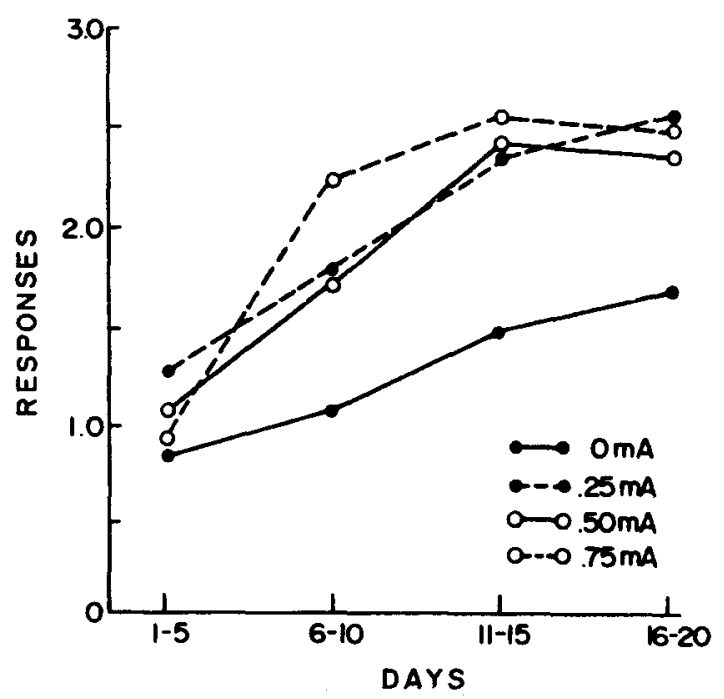

Figure 2. Mean number of responses emitted during the 30-sec CS when preceded by a Shock, of $0, .25, .50$, or .75 $\mathrm{mA}$, shown as a function of 5-day blocks of testing. 
reaction to the previous Shock . For both CS-Shock $_{2}$ trials and Shock -CS-Shock $_{2}$ trials, an identical signal for shock preceded an identical shock. Therefore, the CS should have elicited similar levels of conditioned fear in anticipation of shock in both cases. If inhibition of appetitive responding is taken as an index of fear, the animals should have exhibited similar rates of responding to the CS when the CS was preceded by Shock ${ }_{1}$ and when it was not. The finding that Shock $_{1}$ at all three intensities was followed by increased responding during the CS suggests that the bursts were not due to the presence after Shock, of a reliable signal for safety, but constituted a reaction to shock itself. Thus, unless it is assumed that Shock $_{1}$ served as a discriminative cue for distinguishing safety from danger during the subsequent CS, the data from this study rule out a safety signal explanation in a manner that is not predicated on assumptions regarding a subject's learning that a schedule of aversive events is temporally random.

While the present study suggests that postshock bursts can be a reaction to shock and may not be due to a subject's anticipation of forthcoming shock, the question remains as to whether the bursts were caused by the aversive properties of shock as opposed to the suddenness of shock (Church et al., 1970). While the hypothesis that postshock increments in responding may result from disinhibition of fear caused by the suddenness of shock was not directly tested in the present study, it nonetheless seems an unlikely explanation. First, it should be noted that both CS-Shock ${ }_{2}$ and Shock ${ }_{1}$-CS-Shock ${ }_{2}$ were introduced by a sudden and salient stimulus-Shock ${ }_{1}$ in one case and the 70-dB tone in the other. Second, Figure 2 shows that during the first 10 days of the experiment, the magnitude of post-Shock ${ }_{1}$ responding increased relative to trials on which Shock was $_{1}$ absent. A disinhibition explanation would seem to predict not a relative increase as a function of trials, but rather a decrease.

Assuming that the postshock increase in responding was caused by the aversive properties of shock, one may ask whether that increase was due to a reflexive activation of responding such that the relative rate of responding was no longer indicative of the subject's level of conditioned fear. In this case, Shock, may have produced not a subsequent release from fear but a short-term increment in general arousal or emotionality which acted to energize barpressing for food. Since the withholding of a usual reward has been usefully employed as an aversive stimulus and appears to have effects that are similar to those of electric shock (Wagner, 1969), it may be that these data reflect a frustration effect resembling that inferred from the situation in which an animal runs faster in a second alley after non- reward in the first alley (Amsel, 1958, 1962). However, while such studies raise the possibility here of an interpretation based on arousal after Shock , $_{1}$ it is important to acknowledge that some previous studies that are methodologically more similar to the present one in exploring the effects of electric shock on barpressing are not consistent with a postshock arousal phenomenon. As Myer (1971) has noted, the bulk of evidence (e.g., Bevan, Bell, \& Lankford, 1967; Strongman, 1967) indicates that preshock does not facilitate but suppresses instrumental responding. Therefore, the extent to which an arousal interpretation applies to the current data seems unclear.

If, however, one continues to assume that the magnitude of response suppression varies with the magnitude of conditioned fear, then it may be that subjects experienced less fear during the CS when it was preceded by a Shock ${ }_{1}$. This suggests that after the termination of an aversive event there exists a positive motivational state. Such a positive state would have reduced some of the fear during the CS, resulting in an increase in appetitive responding. Solomon and Corbit (1974) have proposed a theory that posits the presence of a positive state after the termination of an aversive event. Their opponentprocess theory of motivation suggests that a certain hedonic process, called an a-process, is aroused by its adequate stimulus and remains active until that stimulus is terminated. A second process that is opposite in hedonic value is also activated. This b-process is sluggish in nature: it appears after the onset of the a-process, and decays after the stimulus and the a-process have terminated. The animal's hedonic state is the sum, [a-b]. Further, the theory holds that repeated presentations of the same motivating stimulus have no effect upon the a-process. However, after many presentations, the b-process is strengthened in intensity. Thus, during stimulus onset, the strenghtened b-process works to diminish the magnitude of the A state. When the stimulus and the A state are terminated, the strengthened B state is apparent in terms of behavior that is motivationally more intense than before.

The present finding of postshock bursts in appetitive responding is consistent with the expectations of the opponent-process theory. Moreover, the finding that post-Shock ${ }_{1}$ responding increased as a function of trials gives added support to an opponentprocess interpretation of the data. These data may indicate the presence of a positive b-process that increased with repeated presentations of Shock . $_{\text {. }}$ Thus, while an analysis of response-burst data in terms of signals for safety may be appropriate in some situations, it does not readily apply to the current study which produced data compatible with opponent-process theory. 


\section{REFERENCES}

AMSEL, A. The role of frustrative nonreward in noncontinuous reward situations. Psychological Bulletin, 1958, 55, 102-119.

AMsEL, A. Frustrative nonreward in partial reinforcement and discrimination training. Psychological Review, 1962, 69, 306-328.

Bevan, W., Bell, R., \& Lankford, H. G. The residual effect of shock upon bar-pressing for water. Psychological Record, 1967, 17, 23-28.

Bolles, R. C. Species-specific defense reactions and avoidance learning. Psychological Review, 1970, 77, 32-48.

Church, R. M., \& GetTy, D. J. Some consequences of a reaction to an aversive event. Psychological Bulletin, 1972, 78, 21-27.

Church, R. W., Wooten, C. L., \& Matrhews, T. J. Contingency between a response and an aversive event in the rat. Journal of Comparative and Physiological Psychology, 1970, 72, 476-485.

DAvis, H. Post-shock responding on appetitive schedules: Aggression or discrimination? Psychonomic Science, 1970, 18, 11-12.

Estes, W. K., \& SkInNer, B. F. Some quantitative properties of anxiety. Journal of Experimental Psychology, 1941, 29, 390-400.

MYER, J. S. Some effects of non-contingent aversive stimulation. In F. R. Brush (Ed.), Aversive conditioning and learning. New York: Academic Press, 1971.
RescorlA, R. A. Probability of shock in the presence and absence of CS in fear canditioning. Journal of Comparative and Physiological Psychology, 1968, 66, 1-5.

SIDMAN, M. Some notes on "bursts" in free operant avoidance experiments. Journal of the Experimental Analysis of Behavior. 1958, 1, 167-172.

Solomon, R. L., \& Corbit, J. D. An opponent-process theory of motivation: I. Temporal dynamics of affect. Psychological Review, 1974, 81, 119-145.

Strongman, K. T. The effect of prior exposure to shock on a visual discrimination by rats. Canadian Joumal of Psychology. $1967,21,57-58$.

UlRich, R. E., \& AzRin, N. H. Reflexive fighting in response to aversive stimulation. Journal of the Experimental Analysis of Behavior, 1962, 5, 511-520.

W AGNER, A. R. Frustrative nonreward: A variety of punishment? In B. A. Campbell \& R. M. Church (Eds.), Punishment and aversive behavior. New York: Appleton-Century-Crofts, 1969.

Weiss, K. M., \& Strongman, K. T. Shock-induced response bursts and suppression. Psychonomic Science, 1969, 15, 238-240.

(Received for publication March 3, 1976; revision accepted June 23,1976 .) 